\title{
Retraction: The Influence of Extensible Archetypes on Machine Learning
}

\author{
Dejian Fang \\ Division of Logistic, Fuqing Branch of Fujian Normal University, \\ Fuqing 350300, China \\ fffdjasina.com
}

Several conference proceedings have been infiltrated by fake submissions generated by the SCIgen computer program. Due to the fictional content the chapter "The Influence of Extensible Archetypes on Machine Learning" by "Dejian Fang" has been retracted by the publisher. Measures are being taken to avoid similar breaches in the future. 